Cahiers $d u$ MONDE RUSSE

\section{Cahiers du monde russe}

Russie - Empire russe - Union soviétique et États indépendants

$47 / 4 \mid 2006$

Varia

\title{
Lynn Mally, Revolutionary Acts | Julie A. Cassiday, The Enemy on Trial | Elizabeth A. Wood. Performing Justice
}

\section{Alexandre Sumpf}

\section{OpenEdition}

\section{Journals}

Édition électronique

URL : https://journals.openedition.org/monderusse/6718

DOI : $10.4000 /$ monderusse. 6718

ISSN : 1777-5388

Éditeur

Éditions de l'EHESS

Édition imprimée

Date de publication : 30 décembre 2006

Pagination : 830-835

ISBN : 978-2-7132-2098-2

ISSN : $1252-6576$

Référence électronique

Alexandre Sumpf, "Lynn Mally, Revolutionary Acts | Julie A. Cassiday, The Enemy on Trial | Elizabeth A. Wood. Performing Justice », Cahiers du monde russe [En ligne], 47/4 | 2006, mis en ligne le 03 juillet 2009, consulté le 03 septembre 2022. URL : http://journals.openedition.org/monderusse/6718; DOI : https://doi.org/10.4000/monderusse.6718

Ce document a été généré automatiquement le 3 septembre 2022.

Tous droits réservés 


\title{
Lynn Mally, Revolutionary Acts | Julie A. Cassiday, The Enemy on Trial | Elizabeth A. Wood. Performing Justice
}

\author{
Alexandre Sumpf
}

\section{RÉFÉRENCE}

Lynn MALLY, Revolutionary Acts. Amateur Theater and the Soviet State, 1917-1938. Ithaca : Cornell University Press, 2000, 250 p.

Julie A. CASSIDAY, The Enemy on Trial. Early Soviet Court on Stage and Screen.

DeKalb : Northern Illinois University Press, 2000, $260 \mathrm{p}$.

Elizabeth A. WOOD. Performing Justice. Agitation Trials in Early Soviet Russia.

Ithaca : Cornell University Press, 2005, $301 \mathrm{p}$.

1 La parution récente de l'ouvrage de l'historienne américaine Elizabeth A. Wood sur les procès d'agitation (agitsudy) s'inscrit dans un champ de recherches renouvelé depuis quelques années. L'agitsud est un élément singulier de la culture soviétique, qui plonge ses racines dans le développement de l'éducation pour les masses de la seconde partie du XIX ${ }^{e}$ siècle et fleurit dans les années 1920. Si E. A. Wood a choisi de l'examiner en tant que tel et d'en brosser l'histoire, Lynn Mally l'aborde en quelques pages serrées, dans le cadre d'une étude plus large du théâtre amateur, sur le plan institutionnel essentiellement. Quant à Julie A.Cassiday, elle tente de démontrer l'aspect fondamentalement théâtral de la justice en Union soviétique.

2 L. Mally interroge le rôle assigné au théâtre dans l'éducation culturelle en Russie/URSS. Le théâtre d'agitation, souligne-t-elle, est un genre qui fait la part belle au théâtre d'amateur en vogue dès le dernier tiers du xix ${ }^{e}$ siècle. Les acteurs professionnels jouent pour de l'argent, devant un large public, et délivrent un message fondé sur des valeurs 
communes et convenues. Au contraire, l'amateur joue pour le plaisir, plutôt en privé, et traite spontanément de thèmes qui posent problème sur un plan local. Le théâtre amateur se déclare contre l'autorité de l'auteur et du metteur en scène, mais aussi contre la passivité du public, car il prône l'ouverture de la troupe aux volontaires de tous niveaux, la création collective du texte, de la mise en scène, des costumes et des décors.

Les bolcheviks, une fois au pouvoir, décident de rénover profondément la pratique théâtrale. Ils rencontrent sur ce point l'adhésion de certains membres du Proletkul't qui conçoivent le théâtre comme un outil révolutionnaire de transformation sociale, et imposent progressivement un changement radical du vocabulaire théâtral. Ainsi, le théâtre populaire (narodnyj) ou amateur (ljubitel'skij) est remplacé par le "théâtre spontané » (samodejatel'nyj). Ce terme, déjà en vogue sous le tsarisme, est chargé d'un nouveau sens par les responsables du Narkompros, V.V. Tihonovič ${ }^{1}$ en tête. Il désigne désormais un théâtre débarrassé de son origine bourgeoise (narodnyj est une étiquette bourgeoise) et de son dilettantisme, pour faire triompher l'activité consciente spontanée de la classe ouvrière. Dans cette perspective, le spectacle devient performance (vystuplenie), l'acteur devient interprète (ispolnitel'), le jeu devient action (dejstvie).

Deux problèmes se posent rapidement : le goût du public ne correspond pas forcément à ce qui est souhaité; l'éducation culturelle spontanée du Proletkul't concurrence les initiatives des instances étatiques désormais en place. La Direction générale de l'Éducation politique (Glavpolitprosvet), chargée de la propagande du communisme auprès de la population adulte, est responsable dès 1920 de la censure des pièces et des spectacles, qu'ils soient de facture classique ou bien prolétariens. Les années 1920 voient le triomphe des "petites formes" théâtrales (malye formy), terme qui désignait auparavant le music-hall et le vaudeville et qui qualifie à présent les agitsudy, les sketches satiriques et les « journaux vivants» (živye gazety).

5 Quoique Mally n'analyse jamais les raisons du choix opéré par les acteurs entre ces différentes " petites formes ", elle les décrit clairement, en insistant sur la mobilisation du public grâce à l'effacement plus ou moins réussi de la frontière entre troupe et spectateurs. Mais cette opération est elle-même rendue difficile, selon Mally, par l'intervention de l'idéologie bolchevique et des instances étatiques. Le drame du théâtre en URSS se joue alors en trois actes: à l'expérimentation des années 1920 succède la "révolution culturelle» menée par les «brigades d'agitation» (agitbrigady) lors du premier plan quinquennal; puis vient la période de stalinisation, c'est-à-dire de professionnalisation forcée de troupes amateur désormais réduites au rang de complément (spatial et temporel) des troupes permanentes.

6 Mally insiste sur la sclérose progressive de la spontanéité et oppose les années 1920 aux années 1930, en retraçant l'histoire de plusieurs troupes connues, comme Sinjaja bluza [Les blouses bleues] ou le TRAM (Teatr rabočej molodeži - Théâtre de la jeunesse ouvrière de Leningrad). Elle reprend donc l'opposition développée en 1978 par Vladimir Papernyj entre «culture 1 » (étalement et dissémination) et «culture 2 » (solidification et hiérarchisation, propre aux années 1930) pour expliquer la soudaine et courte vogue de ces formes de théâtre amateur et spontané. Dans ce contexte, elle ne parvient toutefois pas à établir nettement si, au-delà de la terminologie, l'agitsud a quelque chose à voir avec l'agitbrigada: quid de l'évolution des slogans, de l'apport du théâtre amateur, de la portée politique de ces spectacles?

7 Dans un ouvrage publié la même année - 2000 - et consacré en partie aux procès d'agitation, J. A. Cassiday adopte, quant à elle, une démarche résolument culturelle. 
L'historienne interroge la notion de théâtralité et cherche à l'étendre au champ politique. Elle récuse sans ambages le recours aux archives soviétiques ouvertes depuis 1991 et élit comme source principale la brochure de Rebel'skij, Inscennirovannye sudy. Kak ih organizavat' i provodit' (1926), ainsi que les 130 "procès joués " publiés tout au long des années 1920. Cette approche littéraire de la question nous vaut plusieurs études détaillées et convaincantes, voire agréables à lire.

Cassiday émet l'hypothèse que la justice soviétique est «mythopoiétique » et cherche par conséquent à analyser la tradition soviétique du procès " à spectacle » par le biais de la littérature des " procès joués ». Après la révolution, les nouveaux maîtres de la Russie organisent des procès spectaculaires qui se veulent identiques aux grands procès des révolutionnaires du XIX ${ }^{e}$ siècle. Le premier du genre, en novembre 1918, est intenté à la comtesse Panina ${ }^{2}$. Mais il s'agit d'un procès expéditif et efficace, peu théâtral. De manière plus symptomatique encore, le procès de la famille impériale, en dépit de l'insistance de Trockij, se déroule à huis clos, en province : il n'a rien d'un procès à l'Ancien Régime. Il faut attendre 1922 et l'adoption du nouveau Code pénal pour que les bolcheviks déclenchent véritablement une longue lignée de procès politiques à spectacle : lors du grand procès des socialistes-révolutionnaires, le public est trié sur le volet parmi les militants sûrs, fidèles aux bolcheviks. Pour Cassiday, il s'agit du premier drame légal exemplaire.

En 1928, le procès pour sabotage de Šahty - procès fabriqué, exemplaire et réel à la fois est un épisode pivot qui prépare les grands procès des années de la Terreur. À partir de cette date, littérature et justice " réelle » se trouvent, selon l'auteur, réconciliées dans la récurrence des procès à spectacle - ce qui expliquerait, au passage, la disparition de l'agitsud de la production littéraire. Le chant du cygne du genre se répercute au cinéma. Dans Sekret rapida, tourné en 1930 par P. Dolina, l'ouvrier de la centrale électrique Koloz, alcoolique notoire, assiste en tant que spectateur à son propre procès d'agitation; atterré de ses errements, il entre en scène, repousse l'acteur et modifie le scénario final. Il est réintégré à l'équipe et sa femme revient. Aucun jugement n'est prononcé : ce qui compte ici est la corrélation entre confession, repentance et réintégration - c'est-à-dire la catharsis collective offerte par la représentation théatrale.

Formulons deux réserves majeures. Certes, l'ouverture des archives n'est pas une révolution historiographique en soi, mais il paraît aujourd'hui risqué de faire l'économie des documents qu'elles recèlent. C'est à nos yeux la cause de la lacune la plus grave de ce travail - l'absence de contexte historique précis. À la différence de Mally, Cassiday néglige de décrire les processus institutionnels, comme s'ils n'influaient nullement sur la création, et ne mentionne ni publication ni diffusion. En outre, pourquoi éluder la terrible réalité qui précède le procès lui-même: arrestation souvent injuste, interrogatoire où règne torture psychologique et physique? N'est-il jamais venu à l'esprit de ceux qui écrivaient et jouaient ces pièces qu'ils répétaient une farce macabre frappant de plus en plus d'innocents?

11 L'absence de prise en compte de la dimension humaine du sujet, qui intégrerait à la réflexion auteurs, acteurs et spectateurs, ruine une partie de l'argumentation. Ainsi, quand Cassiday relève que les acteurs sont parfois amenés à jouer une partie du public, elle en déduit que les bolcheviks ne font pas confiance à la population, mais qu'en même temps, celle-ci est aisément manipulable! Outre cette contradiction, notons qu'au village ou à l'usine, lieux habituels de l'activité du théâtre amateur, les moindres visages, 
voire les voix de chacun, sont connus et reconnus, ce qui exclut toute intervention extérieure crédible.

Peut-on vraiment comparer procès « réel » et agitsud? Certes, le présupposé idéologique est identique, mais la charge symbolique n'est en aucun cas similaire, puisque condamnation et même reniement sont au bout de l'épreuve judiciaire. Le spectacle, réjouissant peut-être pour une populace antiélitiste qui a reçu sa dose d'agitprop, réside dans le caractère implacable de la sentence. Au contraire, le procès d'agitation est avant tout spectacle, plaidoyer, fondé non sur l'aveu et la condamnation, mais sur une possibilité de rédemption.

C'est d'ailleurs là l'une des conclusions de l'ouvrage éclairant de E. A. Wood, qui aborde pour sa part le procès d'agitation de manière à la fois thématique et chronologique. L'auteur distingue quatre sources convergentes confluant dans l'agitsud, genre qui explose en 1919-1920. La première influence ressortit à la longue tradition chrétienne, orthodoxe en particulier, de "procès de pécheurs ", comme le Procès de l'âme pécheresse (1884) ou le Procès de la vodka (1904). Par ailleurs, la réforme judiciaire de 1864 popularise les procès publics, dont la composante dramatique n'échappe pas aux littérateurs russes. Gor'kij, dans La Mère, livre ainsi son message politique par le biais d'une longue scène de procès. Les éducateurs populaires des années 1890 considèrent volontiers le théâtre comme un outil privilégié de l'éducation culturelle des couches populaires. Sociétés de tempérance ou clubs ouvriers mettent régulièrement en scène des pièces tirées du répertoire classique. Enfin, les avant-gardistes du théâtre et de la littérature font connaître leur mouvement en proposant au public des débats littéraires sous forme de procès : c'est le cas de Brjusov et de Esenin, qui organisent en novembre 1920 le « procès de la poésie contemporaine ».

La guerre civile "catalyse » fortement ce genre théâtral, en quantité et en qualité. La Commission de lutte contre la désertion, créée en décembre 1918, commande ainsi la création du Procès contre le déserteur, joué par exemple le 8 décembre 1920 dans un club ouvrier de la province de Smolensk. Au cours de la guerre civile, les faux procès accusent les ennemis désignés de la Russie rouge, comme Wrangel (texte écrit par D. Furmanov, futur auteur de Čapaev), Poincaré, Wilson. Ils mettent aussi en scène l'Armée rouge ellemême, la classe ouvrière, le parti. Ce sont des spectacles de masse: acteurs nombreux, spectateurs encore plus nombreux qui constituent, aux yeux des agitateurs militaires, un public captif. Le procès d'agitation est défini par Wood comme un spectacle à vertu éducative et participative, dont la fortune serait due à l'ampleur et à la durée de l'entreprise d'acculturation de la population masculine adulte par l'Armée rouge.

Exemple fameux, Le Procès de Lenin a été joué plusieurs fois en 1920 pour un public ouvrier, notamment dans les clubs de cheminots. Dans ce "procès-dispute politique et critique" (političeskij kritičeskij sud-disput), Lenin lui-même, accusé, se voit acquitté et même célébré. Les accusateurs sont à leur tour mis sur la sellette, le délibéré leur renvoie tous les points de l'accusation. Il s'agit ici, par un processus de renversement des rôles et des arguments, de faire triompher bon droit et vérité « naturelle». Ainsi, sous allure de débat, on explique aux ouvriers ce qui se passe dans le pays - et l'on tente de leur faire accepter la nette réduction de leurs avantages en nature et les réformes structurelles de l'industrie. Wood voit dans ce phénomène un rituel de légitimation de la révolution auprès de l'auditoire, tandis que les participants actifs y acquièrent une pratique du discours en public. L'illusion de réel est la condition même de l'illusion de démocratie participative, ressort essentiel du genre de l'agitsud. 
16 Avec la NEP, Wood identifie un changement majeur de thématique et d'objectif, au moment où le genre littéraire se développe de manière spectaculaire. Désormais, à la différence des procès d'agitation datant de la guerre civile, les agitsudy du milieu des années 1920 prennent pour cible non plus des personnalités identifiables, mais des objets, des animaux, des individus stéréotypés. Wood consacre des chapitres distincts aux procès agronomiques (agrosudy), aux procès politiques (politsudy) et au sansud - procès d'agitation sanitaire. Il ressort de cette classification que les agitsudy visent désormais un changement en profondeur du mode de vie (byt) des spectateurs présents en dévoilant au grand jour les conflits sociaux. Enfin, tout comme Mally et Cassiday, Wood associe le reflux plutôt brutal de l'agitsud en tant que genre littéraire à l'irruption de la « révolution culturelle » stalinienne de la fin des années 1920.

Finalement, le tableau de l'agitsud brossé par les trois historiennes se révèle assez incomplet, en particulier du fait que la « réalité » politique, matérielle et quotidienne du média théâtral n'est jamais étudiée de manière approfondie. L'histoire des troupes narrée par Mally peine à proposer une histoire sociale qu'on soupçonne pourtant passionnante. La démarche de Cassiday, qui ambitionne de dévoiler à travers l'agitsud, notamment, la nature profonde du régime soviétique, est invalidée par l'abstraction du sujet de son contexte idéologique, au sens fort du terme. L'ouvrage de Wood, enfin, est utile en tant que typologie d'un genre littéraire très spécial. Mais l'agitsud représente plus que cela: c'est, rappelons-le, un spectacle théâtral, censément amateur, destiné à susciter la participation du public. Mise en scène, jeu, réception ne sont jamais abordés par Wood, sans parler de la capacité même ou de la volonté des cercles dramatiques locaux de s'adonner à une pratique si fortement encouragée par les instances bolcheviques. En d'autres termes, une véritable histoire politique et sociale de l'agitsud et des pratiques théâtrales, surtout ambulantes, reste encore à écrire.

\section{NOTES}

1. Directeur de la section «Théâtre ouvrier et paysan » du Narkompros en 1919, V. V. Tihonovič, activiste de longue date du théâtre populaire, est l'auteur de l'ouvrage Narodnyj teatr, publié en décembre 1917. L'édition révisée de ce livre exposant une vue d'ensemble du théâtre amateur paraît en 1922 sous le titre Samodejatel'nyj teatr.

2. En 1903, Panina avait fondé à Saint-Pétersbourg l'une des Maisons du peuple les plus performantes de Russie. Ministre des Affaires sociales de Kerenskij, elle est inculpée pour avoir refusé de livrer aux bolcheviks les 93000 roubles du coffre-fort de son ministère. 